\title{
S-Generalized Mittag-Leffler Function and its Certain Properties
}

\author{
Praveen Agarwal* , Ayşegül Çetinkaya, Shilpi Jain and İ. Onur Kıymaz
}

\begin{abstract}
In 2014, a new generalized beta function which consist of seven parameters, defined and studied by Srivastava et al. [H. M. Srivastava, P. Agarwal and S. Jain, Generating functions for the generalized Gauss hypergeometric functions, Appl. Math. Comput., 247 (2014), pp. 348-352]. In 2015, Srivastava et al. [H. M. Srivastava, R. Jain and M. K. Bansal, A study of the S-generalized Gauss hypergeometric function and its associated integral transforms, Turkish J. Anal. Number Theory, 3 (2015), pp. 101-104] called this generalization as "S-generalized beta function" and use it to define S-generalized Gauss hypergeometric function. In this paper, by using S-generalized beta function, we introduce a new generalization of Mittag-Leffler function. This new generalization of Mittag-Leffler function is consist of eleven parameters. We also investigate some of its certain properties such as integral representations, recurrence formulas and derivative formulas by using classical and fractional derivatives. Furthermore, we determine its Mellin, beta and Laplace integral transforms.
\end{abstract}

Keywords: Mittag-Leffler function; generalized beta function; fractional derivative; integral transform.

AMS Subject Classification (2010): Primary: 26A33 ; Secondary: 33B15; 33E12; 33C15; 33C20.

${ }^{*}$ Corresponding author

\section{Introduction and Preliminaries}

From the beginning of 20th century, special functions (especially hypergeometric types) attract the great attention of many researchers because of their important role in the study of differential equations describing by different phenomenon. Among these functions, Mittag-Leffler type functions are the most popular ones. Many generalizations of Mittag-Leffler functions have been established and studied by a number of authors in many different ways [2, 3, 5, 6, 8, 14, 20, 22].

Recently, Özarslan and Yılmaz introduced and studied the extended Mittag-Leffler function $E_{\alpha, \beta}^{(\sigma ; \rho)}(z ; p)$ defined by (see, [20, p. 2, Eq. (4)]):

$$
\begin{gathered}
E_{\alpha, \beta}^{(\sigma ; \rho)}(z ; p):=\sum_{n=0}^{\infty} \frac{(\rho)_{n}}{\Gamma(\alpha n+\beta)} \frac{B_{p}(\sigma+n, \rho-\sigma)}{B(\sigma, \rho-\sigma)} \frac{z^{n}}{n !}, \\
(\Re(p) \geq 0, \Re(\rho)>\Re(\sigma)>0)
\end{gathered}
$$

where $B_{p}(x, y)$ is the extended Euler's beta function defined by Chaudhry et al. [10, p. 20, Eq. (1.7)] (see also [11, p. 591, Eq. (1.7)]):

$$
B_{p}(x, y):=\int_{0}^{1} t^{x-1}(1-t)^{y-1} \exp \left(\frac{-p}{t(1-t)}\right) d t, \quad(\Re(p) \geq 0) .
$$

For $p=0,(1.1)$ reduces to Mittag-Leffler function with three parameters defined by Prabhakar [22]

$$
E_{\alpha, \beta}^{\sigma}(z):=\sum_{n=0}^{\infty} \frac{(\sigma)_{n}}{\Gamma(\alpha n+\beta)} \frac{z^{n}}{n !}, \quad(\Re(\alpha)>0),
$$

Received : 17-06-2019, Accepted : 01-08-2019 
and (1.2) reduces to classic beta function [17]

$$
B(x, y)=\int_{0}^{1} t^{x-1}(1-t)^{y-1} d t, \quad(\Re(x)>0, \Re(y)>0) .
$$

In these days, Mittag-Leffler function and numerous of its generalizations get a new area of research due to the close connection with fractional calculus and its applications to the study of differential and integral equations. In the present sequel to the aforementioned and many other recent investigations (see, for example, $[2,3,5,6,8,13-17]$; see also the monograph on the subject of Mittag-Leffler functions by Gorenflo $e t$ al. [12]), we introduce a (presumably new) generalization of Mittag-Leffler function in the following way:

$$
\begin{gathered}
E_{\alpha, \beta}^{(\gamma, \sigma, \tau ; a, b ; \kappa, \mu)}(z ; p):=\sum_{n=0}^{\infty} \frac{(\gamma)_{n}}{\Gamma(\alpha n+\beta)} \frac{B_{p}^{(a, b ; \kappa, \mu)}(\sigma+n, \tau-\sigma)}{B(\sigma, \tau-\sigma)} \frac{z^{n}}{n !} \\
(\Re(p) \geq 0, \Re(\alpha)>0, \Re(\tau)>\Re(\sigma)>0, \min \{\Re(a), \Re(b), \Re(\kappa), \Re(\mu)\}>0),
\end{gathered}
$$

where $B_{p}^{(a, b ; \kappa, \mu)}$ is the S-generalized beta function defined in [27, p. 350, Eq. (1.13)]:

$$
\begin{gathered}
B_{p}^{(a, b ; \kappa, \mu)}(x, y):=\int_{0}^{1} t^{x-1}(1-t)^{y-1}{ }_{1} F_{1}\left(a ; b ; \frac{-p}{t^{\kappa}(1-t)^{\mu}}\right) d t \\
(\Re(p) \geq 0, \min \{\Re(x), \Re(y), \Re(a), \Re(b)\}>0, \min \{\Re(\kappa), \Re(\mu)\}>0) .
\end{gathered}
$$

Clearly, $B_{0}^{(a, b ; \kappa, \mu)}(x, y)=B(x, y), B_{p}^{(a, a ; 1,1)}(x, y)=B_{p}(x, y)$ and finally $B_{p}^{(a, b ; \mu, \mu)}(x, y)=B_{p}^{(a, b ; \mu)}(x, y)$ which defined by Parmar in [21]. For more information about S-generalized beta and the related functions we refer [18, 29] to the readers.

Remark 1.1. Here, it is important to mention that if $p=0$, then

$$
\begin{aligned}
E_{\alpha, \beta}^{(\gamma, \sigma, \tau ; a, b ; \kappa, \mu)}(z ; 0) & =\sum_{n=0}^{\infty} \frac{(\gamma)_{n}(\sigma)_{n}}{(\tau)_{n} n !} \frac{z^{n}}{\Gamma(\alpha n+\beta)} \\
& ={ }_{2}^{\alpha} M_{2}^{\beta}(\gamma, \sigma ; \tau, 1 ; z)
\end{aligned}
$$

where

$$
{ }_{p}^{\alpha} M_{q}^{\beta}\left(a_{1}, \ldots, a_{p} ; b_{1}, \ldots, b_{q} ; z\right)=\sum_{n=0}^{\infty} \frac{\left(a_{1}\right)_{n} \cdots\left(a_{p}\right)_{n}}{\left(b_{1}\right)_{n} \cdots\left(b_{q}\right)_{n}} \frac{z^{n}}{\Gamma(\alpha n+\beta)},(\Re(\alpha)>0)
$$

is called generalized M-series defined in [24, p. 449, Eq. (1)]. Besides,

$$
\begin{aligned}
& E_{\alpha, \beta}^{(\gamma, \sigma, \gamma ; a, b ; \kappa, \mu)}(z ; 0)={ }_{2}^{\alpha} M_{2}^{\beta}(\gamma, \sigma ; \gamma, 1 ; z)=E_{\alpha, \beta}^{\sigma}(z), \\
& E_{\alpha, \beta}^{(\gamma, 1, \gamma ; a, b ; \kappa, \mu)}(z ; 0)={ }_{2}^{\alpha} M_{2}^{\beta}(\gamma, 1 ; \gamma, 1 ; z)=E_{\alpha, \beta}(z), \\
& E_{\alpha, 1}^{(\gamma, 1, \gamma ; a, b ; \kappa, \mu)}(z ; 0)={ }_{2}^{\alpha} M_{2}^{1}(\gamma, 1 ; \gamma, 1 ; z)=E_{\alpha}(z) .
\end{aligned}
$$

And also we have

$$
E_{\alpha, \beta}^{(\rho, \sigma, \rho ; a, a ; 1,1)}(z ; p)=E_{\alpha, \beta}^{(\sigma ; \rho)}(z ; p) .
$$

Throughout the paper we assume $\min \{\Re(a), \Re(b), \Re(\kappa), \Re(\mu)\}>0, \Re(p)>0$, and for the sake of shortness we use $\widehat{E}_{\alpha, \beta}(z ; p)$ instead of $E_{\alpha, \beta}^{(\gamma, \sigma, \tau ; a, b ; \kappa, \mu)}(z ; p)$. Note that, we call (1.4) as S-generalized Mittag-Leffler function.

\section{Main Results}

In this section, we discuss and derive some properties for the S-generalized Mittag-Leffler function defined by (1.4) involving integral representations, integral transforms and fractional derivative properties. Our main results are asserted by theorems below. 
Theorem 2.1. For $\Re(\tau)>\Re(\sigma)>0, \Re(\alpha)>0$, the following integral representation holds true:

$$
\begin{aligned}
\widehat{E}_{\alpha, \beta}(z ; p)= & \frac{1}{B(\sigma, \tau-\sigma)} \int_{0}^{1}\left[t^{\sigma-1}(1-t)^{\tau-\sigma-1}\right. \\
& \left.\times{ }_{1} F_{1}\left(a ; b ; \frac{-p}{t^{\kappa}(1-t)^{\mu}}\right) E_{\alpha, \beta}^{\gamma}(z t)\right] d t .
\end{aligned}
$$

Proof. Using (1.5) in (1.4) and changing the order of integration and summation, we have

$$
\begin{aligned}
\widehat{E}_{\alpha, \beta}(z ; p)= & \frac{1}{B(\sigma, \tau-\sigma)} \int_{0}^{1}\left[t^{\sigma-1}(1-t)^{\tau-\sigma-1}\right. \\
& \left.\times{ }_{1} F_{1}\left(a ; b ; \frac{-p}{t^{\kappa}(1-t)^{\mu}}\right)\left(\sum_{n=0}^{\infty} \frac{(\gamma)_{n}}{\Gamma(\alpha n+\beta)} \frac{(z t)^{n}}{n !}\right)\right] d t
\end{aligned}
$$

which yields the desired integral representation in light of (1.3).

Corollary 2.1. Taking $t=\frac{u}{u+1}$ in (2.1). Then from Theorem 2.1, we have

$$
\begin{aligned}
\widehat{E}_{\alpha, \beta}(z ; p)= & \frac{1}{B(\sigma, \tau-\sigma)} \int_{0}^{\infty}\left[\frac{u^{\sigma-1}}{(u+1)^{\tau}}\right. \\
& \left.\times{ }_{1} F_{1}\left(a ; b ; \frac{-p(u+1)^{\kappa+\mu}}{u^{\kappa}}\right) E_{\alpha, \beta}^{\gamma}\left(\frac{u z}{u+1}\right)\right] d u .
\end{aligned}
$$

Corollary 2.2. Taking $t=\sin ^{2} \theta$ in (2.1). Then from Theorem 2.1, we have

$$
\begin{aligned}
\widehat{E}_{\alpha, \beta}(z ; p)= & \frac{2}{B(\sigma, \tau-\sigma)} \int_{0}^{\pi / 2}\left[(\sin \theta)^{2 \sigma-1}(\cos \theta)^{2 \tau-2 \sigma-1}\right. \\
& \left.\times{ }_{1} F_{1}\left(a ; b ; \frac{-p}{\sin ^{2 \kappa} \theta \cos ^{2 \mu} \theta}\right) E_{\alpha, \beta}^{\gamma}\left(z \sin ^{2} \theta\right)\right] d \theta .
\end{aligned}
$$

Theorem 2.2. For $\Re(\tau)>\Re(\sigma)>0, \Re(\alpha)>0, \Re(s)>0$, the following Mellin transform holds true:

$$
\begin{aligned}
\mathfrak{M}\left\{\widehat{E}_{\alpha, \beta}(z ; p)\right\}= & \frac{\Gamma^{(a, b)}(s) \Gamma(\tau+\mu s-\sigma)}{\Gamma(\gamma) B(\sigma, \tau-\sigma)} \\
& \times{ }_{2} \Psi_{2}\left[\begin{array}{l}
(\gamma, 1),(\sigma+\kappa s, 1) \\
(\beta, \alpha),(\tau+(\kappa+\mu) s, 1)
\end{array} ; z\right] .
\end{aligned}
$$

Proof. Taking the Mellin transform (see e.g. [7]) of S-generalized Mittag-Leffler function, we have

$$
\mathfrak{M}\left\{\widehat{E}_{\alpha, \beta}(z ; p)\right\}=\int_{0}^{\infty} p^{s-1} \widehat{E}_{\alpha, \beta}(z ; p) d p .
$$

By using (2.1) we obtain

$$
\begin{aligned}
\mathfrak{M}\left\{\widehat{E}_{\alpha, \beta}(z ; p)\right\}= & \frac{1}{B(\sigma, \tau-\sigma)} \int_{0}^{\infty}\left[p^{s-1} \int_{0}^{1} t^{\sigma-1}(1-t)^{\tau-\sigma-1}\right. \\
& \left.\times{ }_{1} F_{1}\left(a ; b ; \frac{-p}{t^{\kappa}(1-t)^{\mu}}\right) E_{\alpha, \beta}^{\gamma}(z t) d t\right] d p .
\end{aligned}
$$

Interchanging the order of integration we have

$$
\begin{aligned}
\mathfrak{M}\left\{\widehat{E}_{\alpha, \beta}(z ; p)\right\}= & \frac{1}{B(\sigma, \tau-\sigma)} \int_{0}^{1}\left[t^{\sigma-1}(1-t)^{\tau-\sigma-1} E_{\alpha, \beta}^{\gamma}(z t)\right. \\
& \left.\times \int_{0}^{\infty} p^{s-1}{ }_{1} F_{1}\left(a ; b ; \frac{-p}{t^{\kappa}(1-t)^{\mu}} d p\right)\right] d t .
\end{aligned}
$$


By taking $\frac{p}{t^{\kappa}(1-t)^{\mu}}=u$ in second integral of (2.3) and after simplification, (2.3) can be expressed as

$$
\begin{aligned}
\mathfrak{M}\left\{\widehat{E}_{\alpha, \beta}(z ; p)\right\}= & \frac{\Gamma^{(a, b)}(s)}{B(\sigma, \tau-\sigma)} \\
& \times \int_{0}^{1} t^{\sigma+\kappa s-1}(1-t)^{\tau-\sigma+\mu s-1} E_{\alpha, \beta}^{\gamma}(z t) d t,
\end{aligned}
$$

where $\Gamma^{(a, b)}(s)$ is a specific case $(p=0)$ of the extended gamma function defined by Özergin et al. [19, p. 4603, Eq. 3]:

$$
\begin{gathered}
\Gamma_{p}^{(a, b)}(s)=\int_{0}^{\infty} u^{s-1}{ }_{1} F_{1}\left(a ; b ;-u-\frac{p}{u}\right) d u, \\
(\Re(p) \geq 0, \Re(a)>0, \Re(b)>0, \Re(s)>0) .
\end{gathered}
$$

Considering the definition (1.3) in (2.4) and making some computations, we get

$$
\begin{aligned}
\mathfrak{M}\left\{\widehat{E}_{\alpha, \beta}(z ; p)\right\}= & \frac{\Gamma^{(a, b)}(s) \Gamma(\tau+\mu s-\sigma)}{\Gamma(\gamma) B(\sigma, \tau-\sigma)} \\
& \times \sum_{n=0}^{\infty} \frac{\Gamma(\gamma+n) \Gamma(\sigma+\kappa s+n)}{\Gamma(\alpha n+\beta) \Gamma(\tau+(\kappa+\mu) s+n)} \frac{z^{n}}{n !} .
\end{aligned}
$$

Using the definition of Wright hypergeometric function (see, e.g., [28, p. 21])):

$$
{ }_{p} \Psi_{q}\left[\begin{array}{c}
\left(\alpha_{1}, A_{1}\right), \ldots,\left(\alpha_{p}, A_{p}\right) \\
\left(\beta_{1}, B_{1}\right), \ldots,\left(\beta_{q}, B_{q}\right)
\end{array} ; z\right]=\sum_{k=0}^{\infty} \frac{\prod_{j=1}^{p} \Gamma\left(\alpha_{j}+A_{j} k\right)}{\prod_{j=1}^{q} \Gamma\left(\beta_{j}+B_{j} k\right)} \frac{z^{k}}{k !},
$$

where the coefficients $A_{1}, \ldots, A_{p} \in \mathbb{R}^{+}$and $B_{1}, \ldots, B_{q} \in \mathbb{R}^{+}$satisfying

$$
1+\sum_{j=1}^{q} B_{j}-\sum_{j=1}^{p} A_{j} \geq 0
$$

(2.5) is expressed in terms of ${ }_{2} \Psi_{2}$ function to yield the right-hand side of (2.2).

Corollary 2.3. Taking $s=1$ in (2.2) and $\Gamma^{(a, b)}(s)=\frac{\Gamma(a-s) \Gamma(b) \Gamma(s)}{\Gamma(a) \Gamma(b-s)}$ (see [19]), we have

$$
\begin{aligned}
\int_{0}^{\infty} \widehat{E}_{\alpha, \beta}(z ; p) d p= & \frac{\Gamma(a-1) \Gamma(b) \Gamma(\tau+\mu-\sigma)}{\Gamma(a) \Gamma(b-1) \Gamma(\gamma) B(\sigma, \tau-\sigma)} \\
& \times{ }_{2} \Psi_{2}\left[\begin{array}{ll}
(\gamma, 1),(\sigma+\kappa, 1) \\
(\beta, \alpha), & (\tau+\kappa+\mu, 1)
\end{array} ; z\right] .
\end{aligned}
$$

Corollary 2.4. Taking inverse Mellin transform of (2.2), we get the following integral representation

$$
\begin{aligned}
\widehat{E}_{\alpha, \beta}(z ; p)= & \frac{1}{2 \pi i \Gamma(\gamma) B(\sigma, \tau-\sigma)} \int_{\delta-i \infty}^{\delta+i \infty}\left\{\Gamma^{(a, b)}(s) \Gamma(\tau+\mu s-\sigma)\right. \\
& \left.\times{ }_{2} \Psi_{2}\left[\begin{array}{ll}
(\gamma, 1),(\sigma+\kappa s, 1) \\
(\beta, \alpha), & (\tau+(\kappa+\mu) s, 1)
\end{array} ; z\right] p^{-s}\right\} d s .
\end{aligned}
$$

Theorem 2.3. For $\Re(\tau)>\Re(\sigma)>0, \Re(\alpha)>0, \Re(\beta)>0, \Re(\omega)>0$, the following beta transform holds true:

$$
\mathcal{B}\left\{\widehat{E}_{\alpha, \beta}\left(x z^{\alpha} ; p\right) ; \beta, \omega\right\}=\Gamma(\omega) \widehat{E}_{\alpha, \beta+\omega}(x ; p) .
$$

Proof. Using the definition of beta transform (see e.g. [25]) and equation (1.4), we have

$$
\begin{aligned}
& \mathcal{B}\left\{\widehat{E}_{\alpha, \beta}\left(x z^{\alpha} ; p\right) ; \beta, \omega\right\}=\int_{0}^{1} z^{\beta-1}(1-z)^{\omega-1} \widehat{E}_{\alpha, \beta}\left(x z^{\alpha} ; p\right) d z \\
& =\sum_{n=0}^{\infty} \frac{B_{p}^{(a, b ; \kappa, \mu)}(\sigma+n, \tau-\sigma)}{B(\sigma, \tau-\sigma)} \frac{(\gamma)_{n}}{\Gamma(\alpha n+\beta)}\left[\int_{0}^{1} z^{\alpha n+\beta-1}(1-z)^{\omega-1} d z\right] \frac{x^{n}}{n !} \\
& =\sum_{n=0}^{\infty} \frac{B_{p}^{(a, b ; \kappa, \mu)}(\sigma+n, \tau-\sigma)}{B(\sigma, \tau-\sigma)} \frac{(\gamma)_{n}}{\Gamma(\alpha n+\beta)} \frac{\Gamma(\alpha n+\beta) \Gamma(\omega)}{\Gamma(\alpha n+\beta+\omega)} \frac{x^{n}}{n !},
\end{aligned}
$$


which gives the desired result in accordance with (1.4).

Theorem 2.4. For $\Re(\tau)>\Re(\sigma)>0, \Re(\alpha)>0, \Re(\beta)>0, \Re(s)>0$, the following Laplace transform holds true:

$$
\mathcal{L}\left\{z^{\beta-1} \widehat{E}_{\alpha, \beta}\left(x z^{\alpha} ; p\right)\right\}=s^{-\beta} F_{p}^{(a, b ; \kappa, \mu)}\left(\gamma, \sigma ; \tau ; x s^{-\alpha}\right) .
$$

Proof. Applying the Laplace transform (see e.g. $[25,26]$ ) and using $(1.4)$, we have

$$
\begin{aligned}
& L\left\{z^{\beta-1} \widehat{E}_{\alpha, \beta}\left(x z^{\alpha} ; p\right)\right\}=\int_{0}^{\infty} e^{-s z} z^{\beta-1} \widehat{E}_{\alpha, \beta}\left(x z^{\alpha} ; p\right) d z \\
& =\sum_{n=0}^{\infty} \frac{B_{p}^{(a, b ; \kappa, \mu)}(\sigma+n, \tau-\sigma)}{B(\sigma, \tau-\sigma)} \frac{(\gamma)_{n}}{\Gamma(\alpha n+\beta)}\left\{\int_{0}^{\infty} e^{-s z} z^{\alpha n+\beta-1} d z\right\} \frac{x^{n}}{n !} \\
& =\sum_{n=0}^{\infty} \frac{B_{p}^{(a, b ; \kappa, \mu)}(\sigma+n, \tau-\sigma)}{B(\sigma, \tau-\sigma)} \frac{(\gamma)_{n}}{\Gamma(\alpha n+\beta)} \frac{\Gamma(\alpha n+\beta)}{s^{\alpha n+\beta}} \frac{x^{n}}{n !} .
\end{aligned}
$$

Using the definition of S-generalized Gauss hypergeometric function [27, 29]

$$
\begin{gathered}
F_{p}^{(a, b ; \kappa, \mu)}(\gamma, \sigma ; \tau ; z):=\sum_{n=0}^{\infty}(\gamma)_{n} \frac{B_{p}^{(a, b ; \kappa, \mu)}(\sigma+n, \tau-\sigma)}{B(\sigma, \tau-\sigma)} \frac{z^{n}}{n !} \\
(|z|<1 ; \Re(p) \geq 0, \Re(\tau)>\Re(\sigma)>0, \min \{\Re(a), \Re(b), \Re(\kappa), \Re(\mu)\}>0),
\end{gathered}
$$

we obtain the result.

We shall also present the recurrence relation involving the $\widehat{E}_{\alpha, \beta}(z ; p)$.

Theorem 2.5. For $\Re(\tau)>\Re(\sigma)>0, \Re(\alpha)>0$, the following recurrence relation holds true:

$$
\widehat{E}_{\alpha, \beta}(z ; p)=\beta \widehat{E}_{\alpha, \beta+1}(z ; p)+\alpha z \frac{d}{d z} \widehat{E}_{\alpha, \beta+1}(z ; p) .
$$

Proof. Inserting the recurrence relation

$$
E_{\alpha, \beta}^{\gamma}(z)=\beta E_{\alpha, \beta+1}^{\gamma}(z)+\alpha z \frac{d}{d z} E_{\alpha, \beta+1}^{\gamma}(z),
$$

which given in [9], into equation (2.1) we get the result.

Theorem 2.6. The following derivative formulas are valid:

$$
\begin{aligned}
\frac{d^{n}}{d z^{n}}\left\{\widehat{E}_{\alpha, \beta}(z ; p)\right\}= & \frac{(\gamma)_{n}(\sigma)_{n}}{(\tau)_{n}} E_{\alpha, \beta+n \alpha}^{(\gamma+n, \sigma+n, \tau+n ; a, b ; \kappa, \mu)}(z ; p), \\
\frac{d^{n}}{d z^{n}}\left\{z^{\beta-1} \widehat{E}_{\alpha, \beta}\left(\lambda z^{\alpha} ; p\right)\right\}= & z^{\beta-n-1} \widehat{E}_{\alpha, \beta-n}\left(\lambda z^{\alpha} ; p\right), \\
\frac{d^{n}}{d p^{n}}\left\{\widehat{E}_{\alpha, \beta}(z ; p)\right\}= & \frac{(-1)^{n}(a)_{n}}{(b)_{n}} \frac{B(\sigma-n \kappa, \tau-\sigma-n \mu)}{B(\sigma, \tau-\sigma)} \\
& \times E_{\alpha, \beta}^{(\gamma, \sigma-n \kappa, \tau-n \kappa-n \mu ; a+n, b+n ; \kappa, \mu)}(z ; p) .
\end{aligned}
$$

Proof. These formulas can easily obtained by induction on $n$.

We also recall the Riemann-Liouville fractional integral $I_{c+}^{\nu}$ and the fractional derivative $D_{c+}^{\nu}$ (see, e.g., $[17,23]$ )

$$
\left(I_{c+}^{\nu} f\right)(z)=\frac{1}{\Gamma(\nu)} \int_{c}^{z}(z-t)^{\nu-1} f(t) d t,
$$

and

$$
\left(D_{c+}^{\nu} f\right)(z)=\left(\frac{d}{d z}\right)^{n}\left(I_{c+}^{n-\nu} f\right)(z),
$$

where $\nu \in \mathbb{C}, \Re(\nu)>0, n=[\Re(\nu)]+1$. 
Theorem 2.7. Let $c \in \mathbb{R}^{+}=[0, \infty), \nu, \lambda \in \mathbb{C}, \Re(\nu)>0, \Re(\alpha)>0, \Re(\beta)>0, \Re(\tau)>\Re(\sigma)>0$. Then

$$
\begin{aligned}
\left(I _ { c + } ^ { \nu } \left\{(t-c)^{\beta-1}\right.\right. & \left.\left.\widehat{E}_{\alpha, \beta}\left(\lambda(t-c)^{\alpha} ; p\right)\right\}\right)(z) \\
= & (z-c)^{\beta+\nu-1} \widehat{E}_{\alpha, \beta+\nu}\left(\lambda(z-c)^{\alpha} ; p\right)
\end{aligned}
$$

and

$$
\begin{aligned}
\left(D _ { c + } ^ { \nu } \left\{(t-c)^{\beta-1}\right.\right. & \left.\left.\widehat{E}_{\alpha, \beta}\left(\lambda(t-c)^{\alpha} ; p\right)\right\}\right)(z) \\
& =(z-c)^{\beta-\nu-1} \widehat{E}_{\alpha, \beta-\nu}\left(\lambda(z-c)^{\alpha} ; p\right)
\end{aligned}
$$

are valid.

Proof. By making use of (2.6) and (1.4) and applying term-by-term fractional integration by virtue of the formula (see, e.g., [23])

$$
\begin{gathered}
\left(I_{c+}^{\nu}\left\{(t-c)^{\beta-1}\right\}\right)(z)=\frac{\Gamma(\beta)}{\Gamma(\beta+\nu)}(z-c)^{\beta+\nu-1}, \\
(\beta, \nu \in \mathbb{C}, \Re(\beta)>0, \Re(\nu)>0)
\end{gathered}
$$

we have

$$
\begin{aligned}
& \left(I_{c+}^{\nu}\left\{(t-c)^{\beta-1} \widehat{E}_{\alpha, \beta}\left(\lambda(t-c)^{\alpha} ; p\right)\right\}\right)(z) \\
& =\sum_{k=0}^{\infty} \frac{(\gamma)_{k}}{\Gamma(\alpha k+\beta)} \frac{B_{p}^{(a, b ; \kappa, \tau)}(\sigma+k, \tau-\sigma)}{B(\sigma, \tau-\sigma)} \frac{\lambda^{k}}{k !}\left(I_{c+}^{\nu}\left\{(t-c)^{\beta+\alpha k-1}\right\}\right)(z) \\
& =(z-c)^{\beta+\nu-1} \sum_{k=0}^{\infty} \frac{(\gamma)_{k}}{\Gamma(\alpha k+\beta+\nu)} \frac{B_{p}^{(a, b ; \kappa, \tau)}(\sigma+k, \tau-\sigma)}{B(\sigma, \tau-\sigma)} \frac{\left(\lambda(z-c)^{\alpha}\right)^{k}}{k !} .
\end{aligned}
$$

Again using (1.4), we are led to the desired result (2.8) of Theorem 2.7.

Next, taking into account (1.4), (2.7), (2.8) and the second equality of Theorem 2.6, we have

$$
\begin{aligned}
\left(D _ { c + } ^ { \nu } \left\{(t-c)^{\beta-1}\right.\right. & \left.\left.\widehat{E}_{\alpha, \beta}\left(\lambda(t-c)^{\alpha} ; p\right)\right\}\right)(z) \\
& =\left(\frac{d}{d z}\right)^{n}\left(I_{c+}^{n-\nu}\left\{(t-c)^{\beta-1} \widehat{E}_{\alpha, \beta}\left(\lambda(t-c)^{\alpha} ; p\right)\right\}\right)(z) \\
& =\left(\frac{d}{d z}\right)^{n}\left\{(z-c)^{\beta+n-\nu-1} \widehat{E}_{\alpha, \beta+n-\nu}\left(\lambda(z-c)^{\alpha} ; p\right)\right\} \\
& =(z-c)^{\beta-\nu-1} \widehat{E}_{\alpha, \beta-\nu}\left(\lambda(z-c)^{\alpha} ; p\right)
\end{aligned}
$$

which gives (2.9).

Now, we shall discuss about extended Riemann-Liouville and Caputo fractional derivatives related to the $\widehat{E}_{\alpha, \beta}(z ; p)$ given by (1.4). Recently, Agarwal et al [4] and Agarwal and Agarwal [1] introduced the extended Riemann-Liouville and Caputo fractional derivative operators respectively.

Definition 2.1. The extended Riemann-Liouville fractional derivative is defined as [4]:

$$
\mathcal{R}_{z}^{\nu, p ; \kappa, \mu} f(z)=\frac{1}{\Gamma(-\nu)} \int_{0}^{z}(z-t)^{-\nu-1} f(t){ }_{1} F_{1}\left(a ; b ; \frac{-p z^{\kappa+\mu}}{t^{\kappa}(z-t)^{\mu}}\right) d t
$$

where $\Re(p)>0, \Re(\kappa)>0, \Re(\mu)>0$ and $\Re(\nu)<0$. 
Definition 2.2. The extended Caputo fractional derivative is defined as [1]:

$$
\begin{aligned}
\mathcal{C}_{z}^{\nu, p ; \kappa, \mu} f(z)= & \frac{1}{\Gamma(m-\nu)} \int_{0}^{z}\left[(z-t)^{m-\nu-1}\right. \\
& \left.\times{ }_{1} F_{1}\left(a ; b ; \frac{-p z^{\kappa+\mu}}{t^{\kappa}(z-t)^{\mu}}\right) \frac{d^{m}}{d t^{m}} f(t)\right] d t,
\end{aligned}
$$

where $\Re(p)>0, \Re(\kappa)>0, \Re(\mu)>0, m-1<\Re(\nu)<m, m \in \mathbb{N}$.

In the case $p=0,(2.10)$ and (2.11) becomes classical Riemann-Liouville and classical Caputo fractional derivatives, respectively.

Theorem 2.8. For $\Re(\tau)>\Re(\sigma)>0, \Re(\alpha)>0$, the following formula holds true:

$$
\mathcal{R}_{z}^{\sigma-\tau, p ; \kappa, \mu}\left\{z^{\sigma-1} \mathrm{E}_{\alpha, \beta}^{\gamma}(z)\right\}=z^{\tau-1} \frac{\Gamma(\sigma)}{\Gamma(\tau)} \widehat{E}_{\alpha, \beta}(z ; p) .
$$

Proof. Using (2.10) by replacing $\nu$ by $\sigma-\tau$ and applying on $z^{\sigma-1} \mathrm{E}_{\alpha, \beta}^{\gamma}(z)$, we obtain

$$
\begin{aligned}
\mathcal{R}_{z}^{\sigma-\tau, p ; \kappa, \mu}\left\{z^{\sigma-1} E_{\alpha, \beta}^{\gamma}(z)\right\}= & \frac{1}{\Gamma(\tau-\sigma)} \int_{0}^{z}\left[(z-t)^{\tau-\sigma-1}\right. \\
& \left.\times{ }_{1} F_{1}\left(a ; b ; \frac{-p z^{\kappa+\mu}}{t^{\kappa}(z-t)^{\mu}}\right) t^{\sigma-1} E_{\alpha, \beta}^{\gamma}(t)\right] d t .
\end{aligned}
$$

Taking $t=u z$ and considering integral representation (2.1), we get

$$
\begin{aligned}
\mathcal{R}_{z}^{\sigma-\tau, p ; \kappa, \mu}\left\{z^{\sigma-1} E_{\alpha, \beta}^{\gamma}(z)\right\} & =\frac{z^{\tau-1}}{\Gamma(\tau-\sigma)} \int_{0}^{1}\left[u^{\sigma-1}(1-u)^{\tau-\sigma-1}\right. \\
& \left.\times{ }_{1} F_{1}\left(a ; b ; \frac{-p}{u^{\kappa}(1-u)^{\mu}}\right) E_{\alpha, \beta}^{\gamma}(u z)\right] d u \\
& =\frac{z^{\tau-1}}{\Gamma(\tau-\sigma)} B(\sigma, \tau-\sigma) \widehat{E}_{\alpha, \beta}(z ; p),
\end{aligned}
$$

which gives (2.12).

Theorem 2.9. For $m-1<\Re(\sigma-\tau)<m<\Re(\sigma), \Re(\alpha)>0$, the following formula holds true:

$$
\mathcal{C}_{z}^{\sigma-\tau, p ; \kappa, \mu}\left\{z^{\sigma-1} \mathrm{E}_{\alpha, \beta}^{\sigma-m}(z)\right\}=z^{\tau-1} \frac{\Gamma(\sigma)}{\Gamma(\tau)} E_{\alpha, \beta}^{(\sigma, \sigma-m, \tau ; a, b ; \kappa, \mu)}(z ; p) .
$$

Proof. Using (2.11) by replacing $\nu$ by $\sigma-\tau$ and applying on $z^{\sigma-1} \mathrm{E}_{\alpha, \beta}^{\sigma-m}(z)$, we get

$$
\begin{aligned}
\mathcal{C}_{z}^{\sigma-\tau, p ; \kappa, \mu}\left\{z^{\sigma-1}\right. & \left.E_{\alpha, \beta}^{\sigma-m}(z)\right\}=\frac{1}{\Gamma(m+\tau-\sigma)} \int_{0}^{z}\left[(z-t)^{m+\tau-\sigma-1}\right. \\
& \left.\times{ }_{1} F_{1}\left(a ; b ; \frac{-p z^{\kappa+\mu}}{t^{\kappa}(z-t)^{\mu}}\right) \frac{d^{m}}{d t^{m}}\left\{t^{\sigma-1} E_{\alpha, \beta}^{\sigma-m}(t)\right\}\right] d t \\
= & \frac{1}{\Gamma(m+\tau-\sigma)} \frac{\Gamma(\sigma)}{\Gamma(\sigma-m)} \int_{0}^{z}\left[t^{\sigma-m-1}(z-t)^{m+\tau-\sigma-1}\right. \\
& \left.\times{ }_{1} F_{1}\left(a ; b ; \frac{-p z^{\kappa+\mu}}{t^{\kappa}(z-t)^{\mu}}\right) E_{\alpha, \beta}^{\sigma}(t)\right] d t .
\end{aligned}
$$

Taking $t=u z$ and considering integral representation (2.1), we have

$$
\begin{aligned}
\mathcal{C}_{z}^{\sigma-\tau, p ; \kappa, \mu} & \left\{z^{\sigma-1} E_{\alpha, \beta}^{\sigma-m}(z)\right\}=\frac{z^{\tau-1}}{\Gamma(m+\tau-\sigma)} \frac{\Gamma(\sigma)}{\Gamma(\sigma-m)} \\
& \times \int_{0}^{1} u^{\sigma-m-1}(1-u)^{m+\tau-\sigma-1}{ }_{1} F_{1}\left(a ; b ; \frac{-p}{u^{\kappa}(1-u)^{\mu}}\right) E_{\alpha, \beta}^{\sigma}(u z) d u \\
= & \frac{z^{\tau-1} \Gamma(\sigma) B(\sigma-m, m+\tau-\sigma)}{\Gamma(m+\tau-\sigma) \Gamma(\sigma-m)} E_{\alpha, \beta}^{(\sigma, \sigma-m, \tau ; a, b ; \kappa, \mu)}(z ; p) .
\end{aligned}
$$

With little simplification, we obtain (2.13). 


\section{Conclusion}

In our present investigation, we introduced a new generalization of Mittag-Leffler function by using Sgeneralized beta function. The importance of this generalization is that the new function satisfies most of the properties of the original function and provides new relations. In addition, the new generalization ise very compatible with fractional calculus. We concluded our present investigation by remarking that Mittag-Leffler function plays very important role in finding of analytical solutions of the multi-term fractional diffusion equations, that's why the results presented in this paper are very important in application point of view.

Acknowledgment. A special case of S-generalized Mittag-Leffler function (1.4) when $\tau=\gamma$ was presented in International Conference on Recent Advances in Pure and Applied Mathematics (ICRAPAM 2017).

\section{References}

[1] Agarwal R. P. and Agarwal P., Extended Caputo fractional derivative operator, Adv. Stud. Contemp. Math. 25:3 (2015), 301-316.

[2] Agarwal P., Chand M. and Jain S., Certain integrals involving generalized Mittag-Leffler functions, Proc. Nat. Acad. Sci. India Sect. A 85:3 (2015), 359-371.

[3] Agarwal P., Choi J., Jain S. and Rashidi M. M., Certain integrals associated with generalized Mittag-Leffler function, Commun. Korean Math. Soc. 32:1 (2017), 29-38.

[4] Agarwal P., Choi J. and Paris R. B., Extended Riemann-Liouville fractional derivative operator and its applications, J. Nonlin. Sci. Appl. 8:5, (2015), 451-466.

[5] Agarwal P. and Nieto J. J., Some fractional integral formulas for the Mittag-Leffler type function with four parameters, Open Math. 13:1 (2015), 537-546.

[6] Agarwal P., Rogosin S. V. and Trujillo J. J., Certain fractional integral operators and the generalized multi-index Mittag-Leffler functions, Proc. Indian Acad. Sci. Math. Sci. 125:3 (2015), 291-306.

[7] Butzer P.L. and Jansche S., A direct approach to the Mellin transform, J. Fourier Anal. 3 (1997), 325-376.

[8] Camargo R. F., Capelas de Oliveira E. and Vas J., On the generalized Mittag-Leffler function and its application in a fractional telegraph equation, Math. Phys. Anal. Geom. 15:1 (2012), 1-16.

[9] Kurulay M. and Bayram M., Some properties of the Mittag-Leffler functions and their relation with the Wright function, Adv. Differ. Equ. 2012:181 (2012), https:/ / doi.org/10.1186/1687-1847-2012-181.

[10] Chaudhry M. A., Qadir A., Rafique M. and Zubair S. M., Extension of Euler's beta function, J. Comput. Appl. Math. 78 (1997), 19-32.

[11] Chaudhry M. A., Qadir A., Srivastava H. M. and Paris R. B., Extended hypergeometric and confluent hypergeometric functions, Appl. Math. Comput. 159 (2004), 589-602.

[12] Gorenflo R., Kilbas A., Mainardi F. and Rogosin S., Mittag-Leffler functions: Related topics and applications, Springer, Berlin, 2010.

[13] Hilfer R., Fractional time evolution, in: R. Hilfer (Ed.), Applications of Fractional Calculus in Physics, World Scientific Publishing Company, Singapore, New Jersey, London and Hong Kong, 2000.

[14] Hilfer R. and Seybold H., Computation of the generalized Mittag-Leffler function and its inverse in the complex plane, Integral Transform. Spec. Funct. 17 (2006), 637-652.

[15] Kilbas A. A. and Saigo M., On Mittag-Leffler type function, fractional calculus operators and solutions of integral equations, Integral Transform. Spec. Funct. 4 (1996), 355-370.

[16] Kilbas A. A., Saigo M. and Saxena R. K., Generalized Mittag-Leffler function and generalized fractional calculus operators, Integral Transform. Spec. Funct. 15 (2004), 31-49. 
[17] Kilbas A. A., Srivastava H. M. and Trujillo J. J., Theory and Applications of Fractional Differential Equations, North-Holland Mathematical Studies, vol. 204, Elsevier (North-Holland) Science Publishers, Amsterdam, 2006.

[18] Luo M.-J., Milovanovic G. V. and Agarwal P., Some results on the extended beta and extended hypergeometric functions, Applied Mathematics and Computation 248 (2015), 631-651.

[19] Özergin E., Öarslan M. A. and Altın, A. Extension of gamma, beta and hypergeometric function, J. Comput. Appl. Math. 235 (2011), 4601-4610.

[20] Özarslan M. A. and Yılmaz B., The extended Mittag-Leffler function and its properties, J. Inequal. Appl. 2014:85 (2014), https:/ /doi.org/10.1186/1029-242X-2014-85.

[21] Parmar R. K., A new generalization of Gamma, Beta, hypergeometric and confluent hypergeometric functions, Matematiche (Catania) 69 (2013), 33-52.

[22] Prabhakar T. R., A singular integral equation with a generalized Mittag-Leffler function in the kernel, Yokohama Math. J. 19 (1971), 7-15.

[23] Samko S. G., Kilbas A. A. and Marichev O. I., Fractional Integrals and Derivatives. Theory and Applications, Gordon and Breach, Yverdon et al. 1993.

[24] Sharma M. and Jain R., A note on a generalized M-series as a special function of fractional calculus, Fract. Calc. Appl. Anal. 12:4 (2009), 449-452.

[25] Sneddon I. N., The Use of Integral Transforms, Tata McGraw-Hill, New Delhi, 1979.

[26] Spiegel M. R., Theory and Problems of Laplace Transforms, Schaums Outline Series, McGraw-Hill, New York, 1965.

[27] Srivastava H. M., Agarwal P. and Jain S., Generating functions for the generalized Gauss hypergeometric functions, Appl. Math. Comput. 247 (2014), 348-352.

[28] Srivastava H. M. and Karlsson P. W., Multiple Gaussian hypergeometric Series, Ellis Horwood Series: Mathematics and its Applications. Ellis Horwood Ltd., Chichester; Halsted Press [John Wiley \& Sons, Inc.], New York, 1985.

[29] Srivastava H. M., Jain R. and Bansal M. K., A study of the S-generalized Gauss hypergeometric function and its associated integral transforms, Turkish J. Anal. Number Theory 3 (2015), 101-104.

\section{Affiliations}

PraVeEn Agarwal

ADDRESS: Anand International College of Engineering, Jaipur, India.

E-MAIL: goyal.praveen2011@gmail.com

ORCID ID: 0000-0001-7556-8942

AYŞEGÜL ÇETINKAYA

AdDRESS: Department of Mathematics, Ahi Evran University, Kırşehir, Turkey.

E-MAIL: acetinkaya@ahievran.edu.tr

ORCID ID: 0000-0002-1093-5497

SHILPI JAIN

AdDress: Poornima College of Engineering, Jaipur, India.

E-MAIL: shilpijain1310@gmail.com

ORCID ID: 0000-0002-0906-2801 
İ. ONUR KIYMAZ

AdDRESS: Department of Mathematics, Ahi Evran University, Kırşehir, Turkey. E-MAIL: iokiymaz@ahievran.edu.tr

ORCID ID: 0000-0003-2375-0202 